\section{Endotracheal Tube Depth in Premature Newborns}

\section{To the Editor:}

I read the interesting paper by Bartle et al, and there are a few points from the article I would like to discuss. The authors affirmed that appropriate endotracheal tube (ETT) position on chest radiograph in the newborn is approximately at the second or third thoracic vertebrae; I do not completely agree with this statement. In evaluating endotracheal intubation in the neonatal emergency transport setting to establish a nomogram for appropriate ETT depth, my colleagues and I reported ${ }^{2}$ that the mid-carina position corresponds with the ETT tip at the level of the T1-T2 vertebrae. An ETT tip positioned at the $\mathrm{C} 7$ vertebra was defined as being high but still inside the trachea, while an ETT tip positioned at the T3 vertebra was considered as being low but still above the carina. Positioning at either the $\mathrm{C} 7$ or the $\mathrm{T} 3$ vertebra was considered acceptable, while other positions on the chest radiograph were considered incorrect. ${ }^{2}$ I agree with the authors that it is difficult to correctly assess the tip position on chest radiograph; however, it has been reported that if the correct midtrachea position is achieved (ie, T1-T2), neck movements do not significantly influence a safe and correct tube position. ${ }^{3} \mathrm{Al}-$ though our study ${ }^{2}$ described nasal intubation results and Bartle et al evaluated oral intubation, ${ }^{1}$ I believe our observations are relevant to the study by Bartle et al. ${ }^{1}$ Using our parameters to reassess their results could potentially change the authors' conclusions that their weight-based institutional formula has a low sensitivity for predicting proper ETT depth in newborns with very low birth weight.

I partially agree with the authors when they affirmed that ETT depth is poorly correlated with birthweight, and I have stated that estimated tracheal length by weight still remains an approximation. ${ }^{2}$ However, we need to have a reference nomogram to determine the depth of ETT insertion. It is well known in clinical practice that ETT position must be clinically verified following intubation by the presence of symmetrical chest expansion and breath sounds, the absence of loud inspiratory sounds in the epigastric region, and the prompt increase in heart rate and oxygen saturation levels. Bartle and colleagues ${ }^{1}$ came to this conclusion, too. This practice means that slight corrections to the initial tip position are usually performed, at a maximum of $0.5 \mathrm{~cm}$ up or down, and only at a later time after chest radiography. Thus, the use of a nomogram based on the birthweight is certainly useful, and it may be the only viable option as a reference, but caution is recommended in using this reference, especially in very tiny babies.

Carlo Bellini

Neonatal Intensive Care Unit IRCCS Istituto Giannina Gaslini Genoa, Italy

Dr Bellini has disclosed no conflicts of interest.

DOI: $10.4187 /$ respcare.06657

\section{REFERENCES}

1. Bartle RM, Miller AG, Diez AJ, Smith PB, Gentile MA, Puia-Dumitrescu M. Evaluating endotracheal tube depth in infants weighing less than 1 kilogram. Respir Care 2019; 64(3):243-247.

2. Bellini C, Turolla G, De Angelis LC, Calevo MG, Ramenghi LA. Development of a novel reference nomogram for endotracheal intubation in neonatal emergency transport setting. Acta Paediatr 2019;108(1):83-87.

3. Rotschild A, Chitayat D, Puterman ML, Phang MS, Ling E, Baldwin V. Optimal positioning of endotracheal tubes for ventilation of preterm infants. Am J Dis Child Am J Dis Child 1991;145(9):1007-1012.

\section{In Reply:}

We greatly appreciate Dr Bellini's interest in our study ${ }^{1}$ and would like to address his concerns. We agree that the first or second thoracic vertebra (ie, $\mathrm{T} 1$ or $\mathrm{T} 2$ ) has been described in several studies as the reference point for mid-tracheal endotracheal tube (ETT) positioning. ${ }^{2,3}$ The definition of the correct ETT location in our study was "the tip of the ETT below the thoracic inlet and above the carina." The carina is located at T4 in $85 \%$ of infants, with a range of T3-T5 as given by Blayney and Logan. ${ }^{2}$ The "at approximately thoracic vertebrae 2 or 3 " in our methods is for reassuring the reader that we considered the location of the carina in our estimates without specifically defining mid-trachea.

Respiratory movement and head and neck position affect the position of the ETT, and the ETT may move as much as 5-8 mm. ${ }^{4}$ The trachea is shortened during head flexion and during expiration, thus the ETT is pushed toward the carina, whereas the opposite occurs during extension of the head and inspiration. We noted in our paper that "we were unable to determine if there was any neck flexion," without making any other assumptions regarding patient positioning. We respectfully note that, in the study by Bellini et al, ${ }^{5}$ only 12 neonates weighed $<750 \mathrm{~g}$, whereas the mean weight of the 131 subjects in our study was $729 \pm 140$ g. ${ }^{1,5}$ Given the difference in the populations enrolled in each study and that fact that subjects in the study by Bellini et $\mathrm{al}^{5}$ were nasally intubated, it is difficult to compare our results. The determination of ETT depth is challenging in infants with a birth weight $<750 \mathrm{~g}$, and medical practitioners have a tendency to insert the ETT deeper than T2 to avoid the risk of extubation. ${ }^{6}$ Any extension of the infant's head, whether due to the infant's movements, tension from the ventilator circuit, ETT movement due to the securement method, could dislodge the ETT in smaller infants. ${ }^{1}$ This is why the standard practice in our unit is to consider the proper ETT position to be at T2 or T3, which allows a margin of safety.

Our study was a retrospective analysis and included only infants $<1 \mathrm{~kg}$ born at a tertiary center, in a controlled setting, with experienced clinical teams and radiographic evaluation available at all times, and we did not include any intubations done outside our unit or during transport to our hospital, and no infants with a birth weight $>1,000 \mathrm{~g}$. We agree with and recommend clinical evaluation postintubation, including, but not limited to, symmetrical chest rise, equal breath sounds bilaterally, equal response in heart rate and oxygen saturation, and the use of reference nomograms. Based on the available data from our study and others, ${ }^{7,8}$ weight-based methods for determining initial ETT depth may provide a starting point, but ETT depth should be adjusted based on clinical assessment, and chest radiography should be performed as quickly as possible.

\section{Renee M Bartle Andrew G Miller Michael A Gentile Brian P Smith Durham, North Carolina} Mihai Puia-Dumitrescu Duke University Medical Center

Dr Miller has disclosed a relationship with VOCSN. The other authors have disclosed no conflicts of interest.

DOI: $10.4187 /$ respcare.06887 